\title{
HARDY INEQUALITIES FOR SOME NON-CONVEX DOMAINS
}

\section{WALEED ABUELELA}

Abstract. Considering two different geometrical conditions, we obtain some new Hardy-type inequalities for non-convex domains in $\mathbb{R}^{n}$. In order to do so, we study the three-dimensional case and then generalise the approach to the $n$-dimensional case.

Mathematics subject classification (2010): 26Dxx, 26D07, 26D10, 34A40.

Keywords and phrases: Hardy inequality, non-convex domains.

\section{REFERENCES}

[1] A. AnCona, On strong barriers and an inequality of Hardy for domains in $\mathbb{R}^{n}$, J. London Math. Soc., 34(2): 274-290, (1986.)

[2] A. Balins Ky, A. Laptev And A. V. Sobolev, Generalized Hardy inequality for magnetic Dirichlet forms, J. Statistical Physics, 116: 507-521, (2004).

[3] M. SH. BIRMAN AND A. LAPTEV, The negative discrete spectrum of a two-dimensional Schrödinger operator, Comm. Pure. Appl. Math., XLIX: 967-997, (1996).

[4] E. B. DAvies, Heat kernels and spectral theory, Cambridge University Press, (1989).

[5] E. B. DAvies, Spectral theory and differential operators, Cambridge University Press, (1995).

[6] E. B. Davies, The Hardy constant, Quart. J. Math. Oxford, 46(2): 417-431, (1995).

[7] E. B. DAVIES, A review of Hardy inequalities, The Maz'ya anniversary collection, Vol. 2, oper. Theory Adv. Appl. Birkhäuser, Basel, 110: 55-67, (1999).

[8] G. H. Hardy, J. E. Littlewood and G. Polya, Inequalities, Cambridge University Press, first edition, (1934).

[9] A. Laptev And A. V. Sobolev, Hardy inequalities for simply connected planar domains, Amer. Math. Soc. Trans. Ser. 2, 255: 133-140, (2008).

[10] J. Tidblom, Improved $L^{p}$ Hardy inequalities, PhD thesis, Stockholm University, (2005). 